\title{
Leaflet Fracture and Embolization of a CarboMedics Prosthetic Mitral Valve: Case Report
}

\author{
Tae Yeon Kim, M.D., Myoung Young Kim, M.D. \\ Department of Thoracic and Cardiovascular Surgery, Samsung Changwon Hospital, Sungkyunkwan University School of Medicine, Changwon, Korea
}

\author{
ARTICLE INFO \\ Received May 13, 2020 \\ Revised October 13, 2020 \\ Accepted October 18, 2020 \\ Corresponding author \\ Myoung Young Kim \\ Tel 82-55-233-8899 \\ Fax 82-55-233-8899 \\ E-mail kmy1217@hanmail.net \\ ORCID \\ https://orcid.org/0000-0002-0967-8876 \\ TThis manuscript was presented at the 2 nd \\ Busan-Ulsan-Gyeongnam conference, in \\ Busan, Republic of Korea, June 7, 2018.
}

\begin{abstract}
Fracture of prosthetic valve leaflets in the absence of traumatic injury is very rare. Leaflet fracture can cause acute pulmonary edema and cardiogenic shock and is potentially life-threatening, requiring emergency surgery. Thus, a leaflet fracture must be diagnosed quickly and accurately. We present the case of a 46-year-old man with CarboMedics prosthetic aortic and mitral valve replacements implanted 24 years previously. The patient presented at our emergency department with abrupt dyspnea and fever. We diagnosed severe mitral valve regurgitation with anterior leaflet fracture. The patient underwent venoarterial extracorporeal membrane oxygenation and delayed mitral valve replacement. The foreign body was removed step by step because the diagnosis was missed. Two pieces of broken leaflets were found in the left common iliac artery and left external iliac artery. The patient was treated successfully and remains asymptomatic 1 year following surgery.
\end{abstract}

Keywords: Mitral valve replacement, Leaflet fracture, Cardiogenic shock, Case report

\section{Case report}

A 43-year-old male patient underwent aortic valve and mitral valve replacement with CarboMedics valves (Sultzer CarboMedics Inc., Austin, TX, USA) at a university hospital in 1994 and subsequently took warfarin. Wisdom teeth were extracted without prophylactic antibiotics in early 2018 at a follow-up visit. The patient experienced coughing and sputum for several days before visiting the emergency room. In addition, the patient experienced generalized weakness, deteriorated fever, and shortness of breath at noon on the day of the emergency room visit. When he arrived at the local hospital, endotracheal intubation was performed immediately because his oxygen saturation was $68 \%$. Transthoracic echocardiography showed normal left ventricular ejection and left atrial dilatation was confirmed. However, the vegetation and valve function of the aortic and mitral mechanical valves could not be evaluated at that time. The patient was transferred to our hospital due to uncontrolled pulmonary edema.

All lung zones were auscultated with crackle sounds. At the emergency room examination, the patient's blood pressure was $110 / 90 \mathrm{~mm} \mathrm{Hg}$, the pulse was 169 beats/min, the respiratory rate was 35 breaths/min, the body temperature was $38.6^{\circ} \mathrm{C}$, and the oxygen saturation was $83 \%$. The following blood lab values were obtained: C-reactive protein, $54.6 \mathrm{mg} / \mathrm{L}$; white blood cell count, $32,820 / \mu \mathrm{L}$; procalcitonin, $3.21 \mathrm{ng} / \mathrm{mL}$; creatine kinase-MB, $2.6 \mathrm{ng} / \mathrm{mL}$; troponin-I, $0.276 \mathrm{ng} / \mathrm{mL}$; pro-brain natriuretic peptide, $12,454 \mathrm{pg} / \mathrm{mL}$; D-dimer, $493 \mathrm{ng} / \mathrm{mL}$; international normalized ratio, 1.71; blood urea nitrogen, $34 \mathrm{mg} / \mathrm{dL}$; and creatinine, $3.2 \mathrm{mg} / \mathrm{dL}$. Meropenem and vancomycin were administered for suspected infectious endocarditis and pneumonia as a cause of fever. Hypoxia caused by acute respiratory distress syndrome worsened and the patient's blood pressure was not maintained with vasopressors. To evaluate the cause of cardiogenic shock, we performed coronary angiography.

On coronary angiography, only 1 leaflet was found in the mitral valve, but the diagnosis of mitral valve regurgitation due to leaflet fracture was missed (Fig. 1). On the second day of hospitalization, transthoracic echocardiography was performed. We found no vegetation on the aortic and mitral valves and the function of the aortic valve was good. However, 1 of the leaflets of the mitral valve was not seen and severe mitral regurgitation was confirmed. On the third day of hospitalization, the thoracic surgery de- 
partment was contacted because mobility of the mitral valve leaflet could not be observed with transesophageal echocardiography (Fig. 2). Subsequently, an emergency redo-mitral valve replacement $(29 \mathrm{~mm}$; St. Jude Medical Inc., St. Paul, MN, USA) was performed.

In the operating room, no vegetation of the mitral valve was observed, but a single leaflet was completely separated from the existing CarboMedics mechanical valve (Fig. 3A) and the formation of a pannus was severe around the sewing ring. The left atrium, left ventricle, and pulmonary vein were examined using a video endoscope in the operating room, but the missing leaflet could not be found.

After valve replacement surgery, the patient was admitted into the intensive care unit with veno-venous extracorporeal membrane oxygenation due to persistent hypoxia. On the ninth day after surgery, computed tomography of the chest and abdomen was performed to find the missing

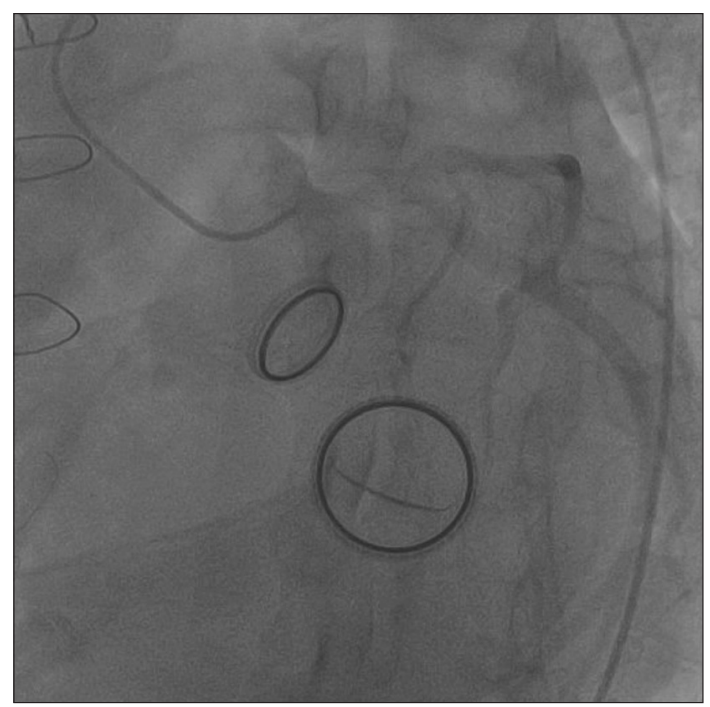

Fig. 1. Preoperative coronary angiography. Only 1 leaflet was observed on the CarboMedics mitral valve. leaflet. A 14.4-mm piece of the valve was found in the left common iliac artery and a $13.9-\mathrm{mm}$ piece was found in the left external iliac artery. On the 23rd day after surgery, the leaflet inside the left external iliac artery was removed via a transverse incision from the mid-inguinal ligament to the lateral side, under general anesthesia. The leaflet fragment in the left common iliac artery was not easy to reach. Therefore, the fragment was removed using a fluoroscopy device (C-arm) in the operating room (Fig. 3B). After 2 operations, the patient was discharged 33 days after hospitalization. The patient underwent outpatient follow-up without specific findings 1 year after surgery.

The study was approved by the Institutional Review Board of Samsung Medical Center (IRB approval no., 202010014). The patient provided written informed consent for the publication of his clinical details and images.

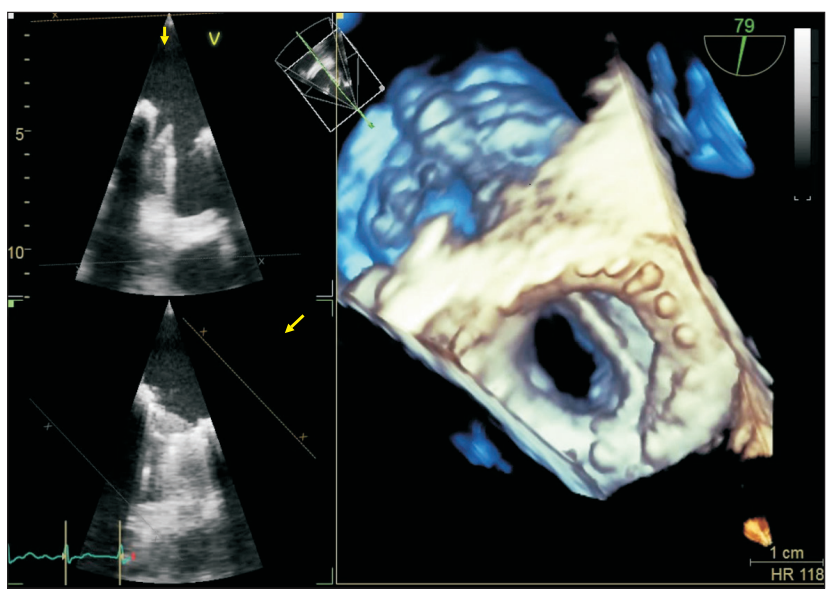

Fig. 2. Preoperative 3-dimensional transesophageal echocardiography. Only 1 leaflet was observed on the CarboMedics mitral valve (arrow).
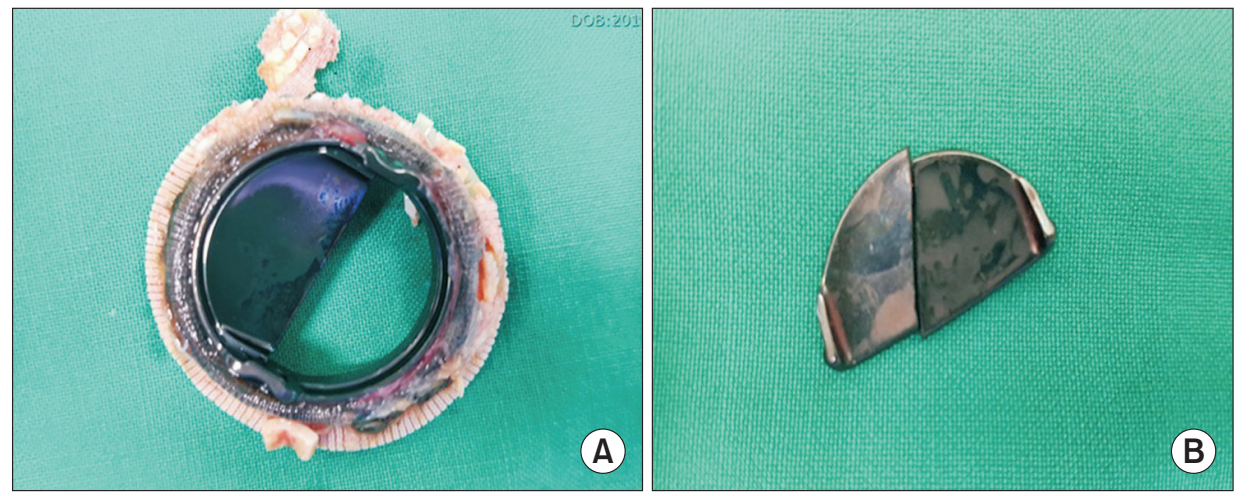

Fig. 3. (A) Remnant of the CarboMedics mitral valve. (B) Two pieces of the broken leaflets. 


\section{Discussion}

Spontaneous leaflet fracture of mechanical heart valves is very rare, but potentially life-threatening. Leaflet fracture usually requires emergency surgery due to acute pulmonary edema and cardiogenic shock. Therefore, a rapid and accurate diagnosis is needed. An examination of preoperative imaging (echocardiography, coronary angiography, chest computed tomography, etc.) is important for patients in whom mechanical valve leaflet fracture is suspected. Transthoracic echocardiography is used for diagnosis and to find missing leaflets, and coronary angiography is also used for the diagnosis [1]. According to Van Steenbergen et al. [2], most fractured leaflets migrate to the abdominal aorta (13\%) or iliac arteries (78\%), and in 1 case migration to the brachiocephalic artery occurred. Wholebody computed tomography is a reliable imaging technique to localize escaped leaflets. Valve leaflet fractures occurred most frequently with the use of Bjork-Shiley convexoconcave products, followed by Edwards-Duromedics products and TRI Technologies products (TRI Technologies Prosthetic Heart Valve; Ltda, Belo Horizonte, Brazil). Valve fracture is very rare in other products [2].

CarboMedics mechanical valves have been used worldwide since December 1986 and have been used clinically since 1988 in Korea [3]. According to the 10-year clinical results of the CarboMedics valve, no cases of death or complications due to structural defects in the valve itself occurred [3]. CarboMedics published a paper on results over 20 years, and the most common cause of reoperation for mitral valve replacement was paravalvular leakage, followed by pannus formation, infective endocarditis, and valve thrombosis. However, there were no cases of reoperation due to structural defects in the valve itself [4]. Similarly, in a study of 330 patients who underwent mitral valve replacement and 142 patients who underwent aortic valve replacement using CarboMedics valves, there were no cases of reoperation due to structural defects in the valve itself [5].

In the present case, echocardiography and coronary an- giography were performed to find the cause of cardiac shock. Even though one of the mitral valve leaflets could not be seen, the rarity of CarboMedics valve fracture was a reason why valve leaflet fracture was not diagnosed and the reoperation was delayed. Based on this case, if a patient who underwent valve replacement surgery complains of sudden respiratory distress and acute pulmonary edema develops, a structural defect in the valve must be suspected to make a rapid diagnosis. In addition, if the patient's systemic condition is good, the missing piece should be located in advance through whole-body computed tomography.

\section{Conflict of interest}

No potential conflict of interest relevant to this article was reported.

\section{ORCID}

Tae Yeon Kim: https://orcid.org/0000-0003-2440-9890

Myoung Young Kim: https://orcid.org/0000-0002-0967-8876

\section{References}

1. Vogel W, Stoll HP, Bay W, Frohlig G, Schieffer H. Cineradiography for determination of normal and abnormal function in mechanical heart valves. Am J Cardiol 1993;71:225-32.

2. Van Steenbergen GG, Tsang QH, van der Heide SM, Verkroost MW, Li WW, Morshuis WJ. Spontaneous leaflet fracture resulting in embolization from mechanical valve prostheses. J Card Surg 2019;34: 124-30.

3. Kim GG, Lee EB, Cho JY, et al. Ten-year clinical experience with CarboMedics valve. Korean J Thorac Cardiovasc Surg 2000;33:64347.

4. Bouchard D, Mazine A, Stevens LM, et al. Twenty-year experience with the CarboMedics mechanical valve prosthesis. Ann Thorac Surg 2014;97:816-23.

5. Santini F, Casali G, Viscardi F, et al. The CarboMedics prosthetic heart valve: experience with 1,084 implants. J Heart Valve Dis 2002; 11:121-6. 